\title{
A scalable self-assembling micellar system for enhanced oral bioavailability and efficacy of lisofylline for treatment of type-I diabetes
}

Kishan S Italiya, Moumita Basak, Samrat Mazumdar, Deepak K Sahel, Richa Shrivastava, Deepak Chitkara, Anupama Mittal*

Department of Pharmacy, Birla Institute of Technology and Science (BITS PILANI), Pilani, Rajasthan, INDIA-333031

\section{Supplementary information}

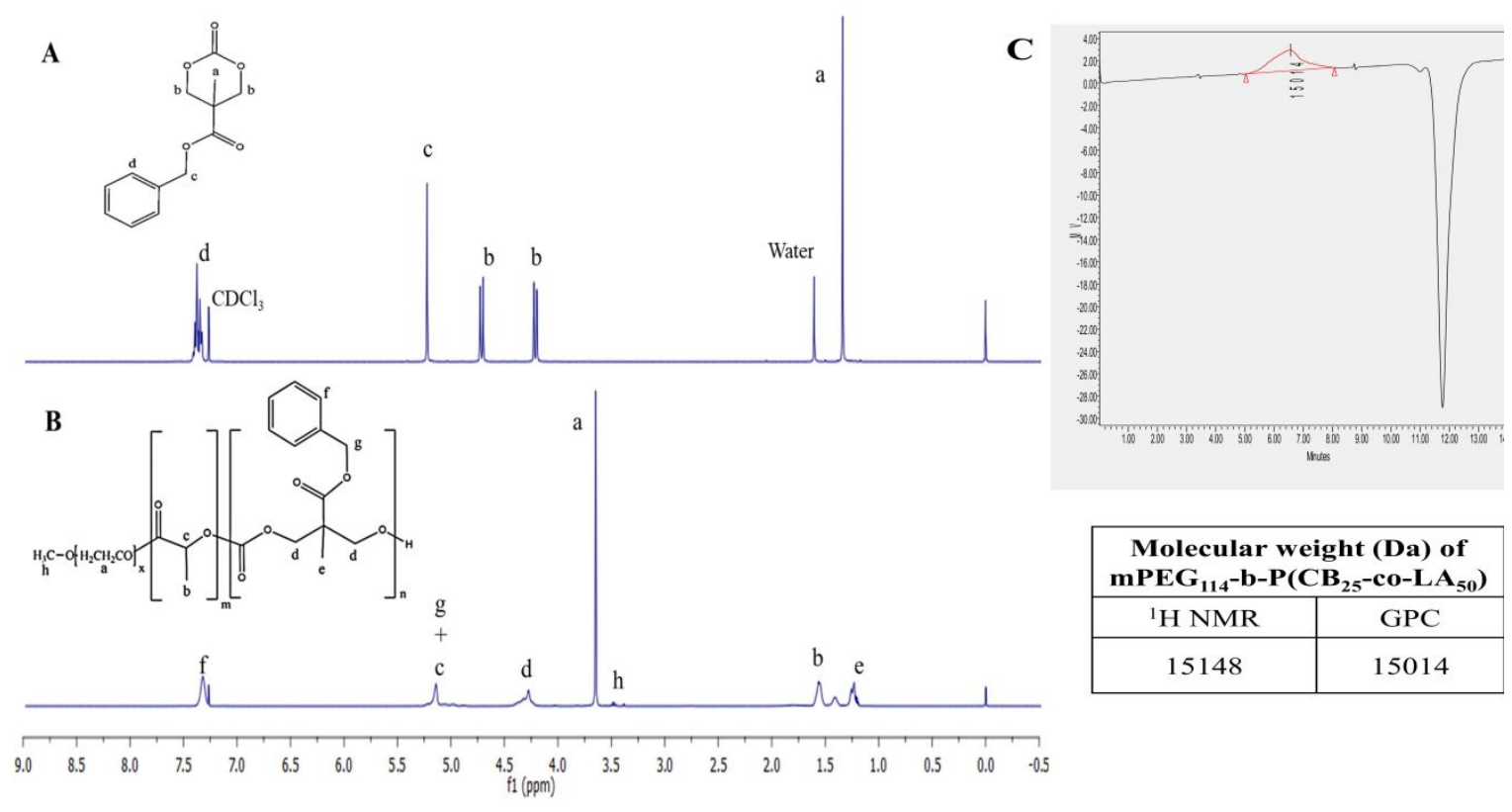

Fig. S1. $m P E G-b-P(C B-c o-L A)$ polymer characterization: (A) and (B) ${ }^{1} \mathrm{H}$ NMR of 5methyl-5-benzyloxycarbonyl-1,3-dioxane-2-one (MBC) monomer and $\mathrm{mPEG-}$ poly(carbonate-co-lactide) polymer respectively and (C) GPC analysis of mPEGpoly(carbonate-co-lactide). 